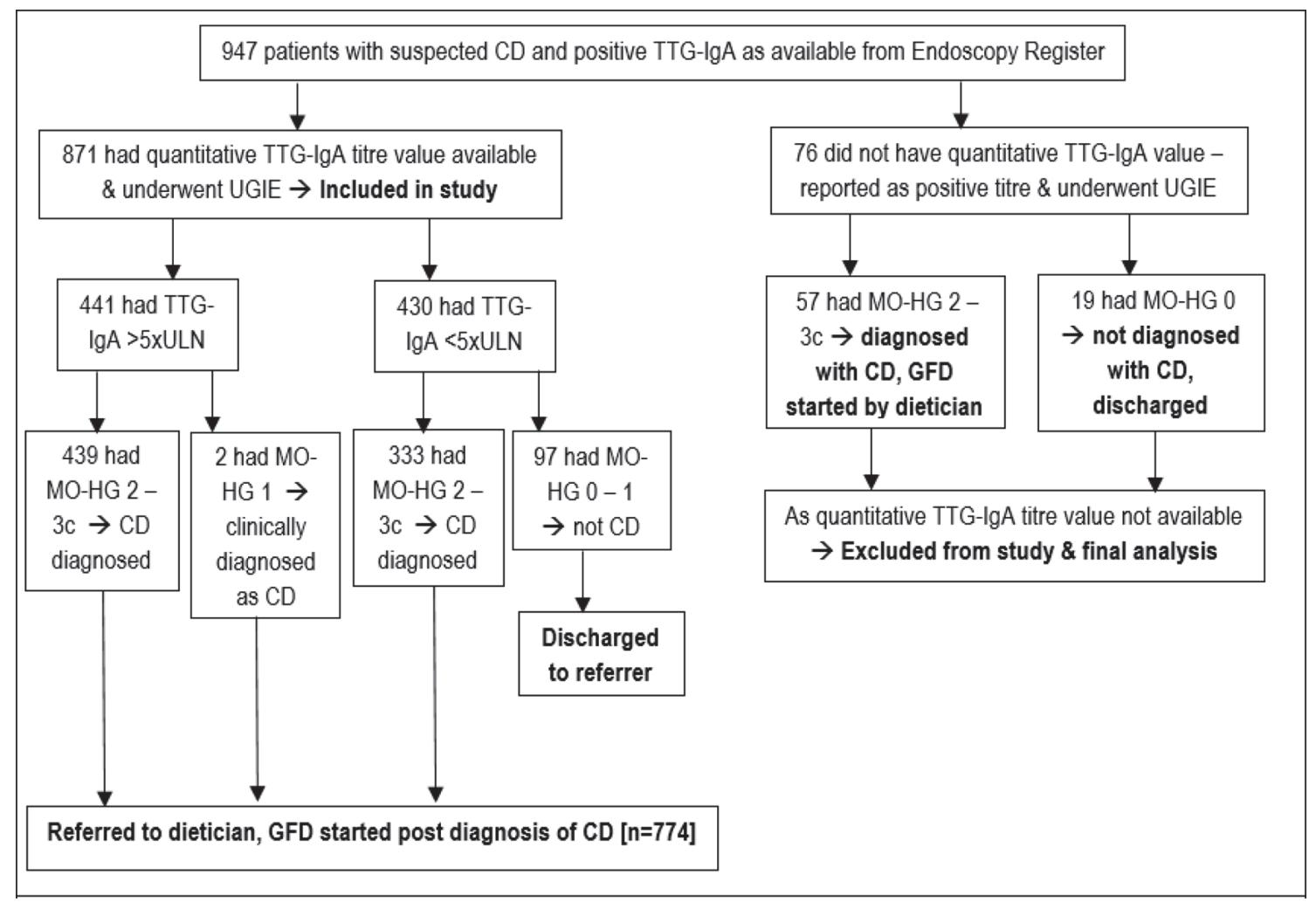

Abstract 08 Figure 1 Flow chart showing TTG-IgA and histological correlation for children with suspected CD.

CD, celiac disease; GFD, gluten free diet; MO-HG, Marsh Oberhuber histological grading; TTG-IgA, IgA-based anti-tissue transglutaminase antibody; UGIE, upper gastrointestinal endoscopy; ULN, upper limit of normal

Aims To explore the relationship of TGA-IgA $\geq 5 \mathrm{x}$ ULN with histological diagnosis of $\mathrm{CD}$ in children referred to a single large tertiary centre.

Methods Prospectively recorded data for children diagnosed with CD following endoscopy over 14-year period (September 2006 to August 2020) was analysed. The data included age, sex, reason for screening, indication for endoscopy, TGA-IgA levels at endoscopy, and histological findings. Where quantitative TGA-IgA was unavailable or not recorded were excluded from the analysis. Statistical analysis was performed using $\chi 2$ analysis and $\mathrm{p}<0.05$ was considered significant.

Results 947 children had endoscopy, but 871 had complete data and were included in final analysis. 772/871 received a histological confirmation of $\mathrm{CD}$ by Marsh-Oberhuber histological grading (MO-HG) 2 to $3 \mathrm{c}$. 441 had TGA-IgA $\geq 5 \mathrm{x}$ and $439(99.5 \%)$ had a positive histological diagnosis. The likelihood of a positive biopsy with TGA-IgA $\geq 5 \mathrm{x}$ titre (439/441) compared to TGA-IgA $<5$ ULN titre (333/430) has strong statistical significance $(\mathrm{p}<0.00001)$. Two children of 441 who had MO-HG $<2$ actually had TGA-IgA $>10$ ULN. The mean and median ages of the patients with confirmed CD $(n=772)$ was 8.68 years and 9.1 years respectively (range $0-17$ years), with a male to female ratio $\approx$ $1: 2$. Figure 1 shows the outcome of the 947 children who had endoscopy.

Conclusion This study showed that $99.5 \%$ of children with TGA-IgA $\geq 5 \times$ ULN had clear histological confirmation of CD with $\mathrm{p}<0.00001$ compared to TGA-IgA $<5 x U L N$. For the same advantages of the current NBP and considering the challenges posed by the COVID-19 pandemic, changing the guidance to TGA-IgA $\geq 5 \times$ ULN appears to be safe and secure for diagnosis of CD in children.

\section{SUSTAINED CLINICAL AND FINANCIAL BENEFITS OF A NUTRITION SUPPORT TEAM}

Michelle Butcher, Tracey Johnson, Gabis Chana, Sue Protheroe, Theo Wong, Wolfram Haller. Birmingham Women's and Children's hospital

\subsection{6/flgastro-2021-bspghan.9}

Background The evidence-based standard for optimal nutritional support is the multidisciplinary approach of a Nutrition Support Team (NST). Following a pilot study in 2012 showing reduced usage and wastage of parenteral nutrition $(\mathrm{PN})$ a business case was accepted in 2014 to fund the NST at Birmingham Children's Hospital $(\mathrm{BCH})$. The initial focus of the team was to reduce PN usage and between 2012-2016 we reported a reduction in $\mathrm{PN}$ days of $20 \%$. There was also an increase in the use of standard bags from $6 \%$ to $14.5 \%$ and a reduction in wastage from $5 \%$ to $3 \%$. Cost savings were estimated to exceed $£ 150,000$. Between 2017-2020 the NST has continued to work at reducing $\mathrm{PN}$ use but have actively sourced more standard bags and improved education of clinical teams on appropriate use of standard PN.

Aim To assess whether there has been a sustained reduction in overall PN usage, a further increase in standard bag usage and reduction in wastage between 2017-2020.

Methods PN usage and PN wastage data for 2017-2020 was collated using the $\mathrm{BCH}$ pharmacy database. Data included number of PN referrals, total number of PN days per month, $\%$ standard bags and $\%$ wastage. This was compared with data from 2012-2016. Wastage was defined as unused and discarded PN.

Results Mean PN usage has fallen from 752 PN days/month in 2016 to 634 in 2020 showing a further $15 \%$ reduction in 
the total number of PN bags used (figure 1). This is despite an increase in the mean number PN referrals from 26/month in 2016 to $39 /$ month in 2020 .

Mean\% standard bag usage has increased from $14.5 \%$ in 2016 to $29.5 \%$ in 2020 (figure 2)

There is minimal change in wastage (figure 3) but wastage varied widely between different specialities (surgery: $2.2 \%$, oncology and haematology: $3.6 \%$ and Paediatric Intensive Care (PICU) 7.3\%).

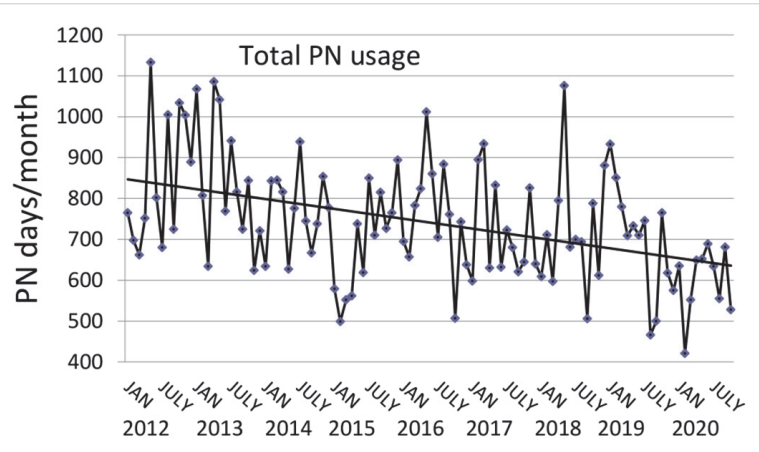

Abstract 09 Figure 1

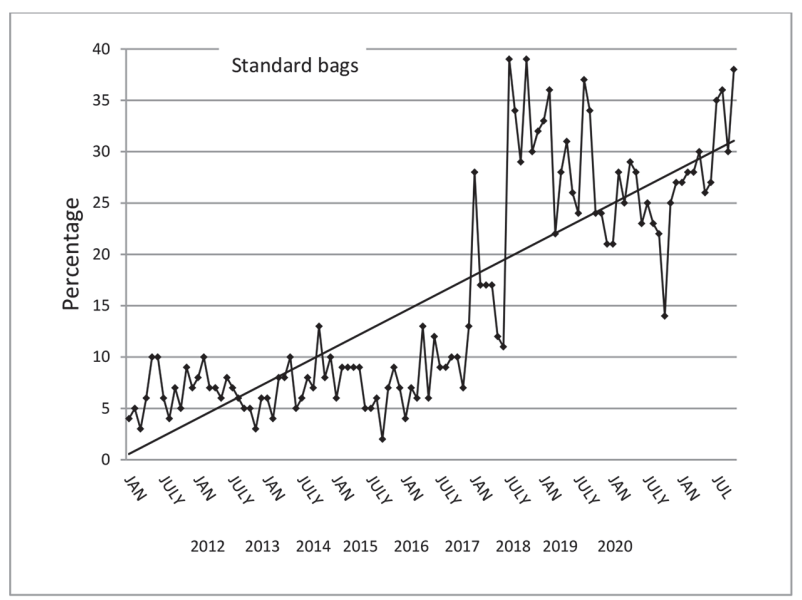

Abstract 09 Figure 2

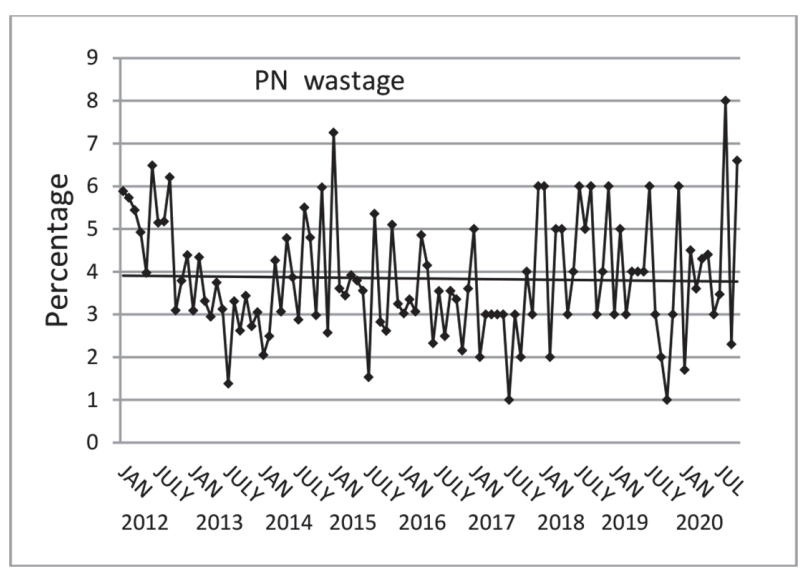

Abstract 09 Figure 3
Summary and conclusion The interventions of the NST have resulted in continued reduction in the number of $\mathrm{PN}$ days despite an increase in referral numbers, suggesting shorter episodes of PN.

A sustained increase in the percentage of standard bags $2017-2020$ is attributed to more frequent consideration of standard PN, particularly when starting and weaning PN. Using clinical expertise the NST has introduced a wider range of standard PN bags. This has also contributed to increased standard PN use without compromising the quality of nutritional support.

There are a number of factors that contribute to reducing PN wastage including using standard bags as these can be recycled. The study has not been able to demonstrate any major impact of standard bag use on overall PN wastage. Wastage is multifactorial and data recording for PN wastage has been optimised to enable thorough evaluation of contributing factors. Most specialities have demonstrated reduced PN wastage, but wastage on PICU remains high. This clinical area will be prioritised for additional NST involvement in 2021.

\section{MAPPING THE CURRENT SERVICE AVAILABILITY IN PAEDIATRIC GASTROENTEROLOGY NETWORKS ACROSS THE UNITED KINGDOM: IS THERE A POSTCODE LOTTERY IN SERVICE PROVISION?}

Sandra Fernandes Lucas, Himadri Chakraborty. Basildon and Thurrock University Hospitals NHS Foundation Trust

\subsection{6/flgastro-2021-bspghan.10}

Background Clinical Networks are formed by Network Centres (NC) and Lead Specialist Centres (LSC) for Gastroenterology and Nutrition to provide high quality, specialist care to the local population. According to the Quality Standards released by RCPCH and BSPGHAN, by definition Network Centres have at least one Consultant Paediatrician with a Special Interest in (SPIN) Paediatric Gastroenterology. It is important to understand the layout of service availability in secondary care and its uniformity across the United Kingdom (UK) to ensure delivery of high-quality care.

Objectives There is scarcity of information about the secondary care network for Paediatric Gastroenterology in the UK. The primary aim of the project was to quantify the secondary care trusts with at least one General Paediatrician with a Special Interest in Gastroenterology and comprehend how these specialists are distributed across the UK. Secondly, we aimed to look at the support these hospitals receive from Lead Specialist Centres in the form of outreach clinics. Finally, the last goal was to map the Paediatric Gastroenterology Clinical Networks in the UK.

Methods The list of hospitals providing Paediatric services in the UK was collected from the National Health Care systems' and Paediatric deaneries' websites for England, Wales, Northern Ireland and Scotland. We identified a number of centres providing Paediatric secondary and tertiary care services, and subsequently contacted them via telephone or e-mail. All the data was collected from Paediatric Doctors (Specialty Registrars or Consultants), Specialist Nurses or Secretaries working in Paediatrics over the period of one year, through standard verbal questionnaire or electronic survey.

Results We identified a total of 153 secondary care trusts providing specialist services to the Paediatric population 